\title{
Aktuelle Forschung zur Körperpsychotherapie, Tanz-, Bewegungstherapie und zu Tanz bei Depression
}

\author{
Iris Bräuninger
}

D er Beitrag beleuchtet neue Erkenntnisse zur Behandlung von Depression. Zunächst wird ein Cochrane Review über Tanz-, Bewegungstherapie vorgestellt, gefolgt von einer Studie zur manualisierten körperpsychotherapeutischen Intervention chronisch depressiver Patienten. Der dritte Artikel stellt eine Interventionsstudie zur Wirksamkeit einer Tanzintervention auf Depression, körperliche Funktion und Behinderungen bei älteren Erwachsenen vor. Abschließend werden Ergebnisse eines Kurzzeittherapie-Programms bei postpartaler Depression präsentiert, welches eine dyadische Mutter-Kind-Gruppenintervention evaluiert.

\section{Wirksamkeit von Tanz-, Bewegungs- therapie bei Depression}

Der Cochrane Review von Meekums und Kolleginnen (2015) überprüfte die Wirksamkeit von Tanz-, Bewegungstherapie bei Depression. Als Cochrane Reviews werden systematische Übersichtsarbeiten verstanden, bei denen alle zu einer therapeutischen Fragestellung relevanten Studien und andere Forschungsergebnisse zusammengefasst werden (Das Cochrane Zentrum 2015). Die Fragestellungen lauteten: Ist Tanz-, Bewegungstherapie effektiver

- als keine Behandlung,

- als eine Standardbehandlung,

als Gesprächstherapien,

- als Medikamente,

- als physikalische Behandlungen wie Tanz oder Sport,

und wie effektiv sind die verschiedenen tanz-, bewegungstherapeutischen Ansätze? Die Datenbanken wurden bis Oktober 2014 nach randomisierten kontrollierten Studien durchsucht. Drei
Studien (147 Teilnehmer) erfüllten die Einschlusskriterien, davon zwei mit erwachsenen Männern und Frauen und eine mit jugendlichen Frauen. Die geringe Studienanzahl und die eingeschränkte Evidenzqualität lassen keine Schlussfolgerungen auf die untersuchten Fragen zu und erlauben keine Aussage für oder gegen die Wirksamkeit von Tanz-, Bewegungstherapie in der Behandlung von Depression. Tanz-, Bewegungstherapie scheint für Erwachsene effektiver als Standardtherapie, wenn auch nicht signifikant. Bei jungen Menschen scheint sie allerdings im Vergleich zur Standardbehandlung nicht wirksamer zu sein.

\section{Manualisierte körperpsychothera- peutische Interventionen bei Patienten mit chronischer Depression}

Daten einer randomisierten Pilotstudie (Röhricht et al. 2013) wurden in einer interpretativen phänomenologischen Sekundäranalyse ausgewertet (Papadopoulos/Röhricht 2014), um die Körpererfahrungen und die emotionalen Reaktionen von chronisch depressiven Patienten auf eine manualisierte körperpsychotherapeutische Intervention zu untersuchen. Hierfür wurden drei Quellen herangezogen: 1. Sitzungsaufzeichnungen, 2. individuelle Patientenberichte (basierend auf 20 Sitzungen) zu Körpererfahrungen, LMAVerhaltensbeobachtungen, Ausdrucksverhalten, Wechsel depressiver Symptome und emotionaler Reaktionsfähigkeit und 3. ein abschließendes halbstrukturiertes zweistündiges Interview mit der Therapeutin über den Therapieprozess. Die Ergebnisse der Datenanalyse weisen darauf hin, dass zu Beginn der Therapie emotionale Distanziertheit, Isolation und nach innen gerichtete Ausdrucksverhalten vorherrschend waren. Hingegen konnten die Teilnehmerlnnen am Ende der 
Intervention Körperhaltung und Gesten mit Empowerment und unterdrückte Wut mit Trauer assoziieren. Ihr Selbstvertrauen, ihre depressive Stimmung und ihre Körperzufriedenheit schienen sich zu verbessern.

\section{Wirksamkeit von Tanz auf Depression, körperliche Funktion und Behinderungen bei älteren Erwachsenen}

Die Machbarkeitsstudie von Murrock und Graor (2014) überprüfte die Wirksamkeit einer Tanzintervention auf Depression, körperliche Funktion und Behinderungen bei älteren Erwachsenen aus einer wirtschaftlich schwachen, innerstädtischen Wohngegend. Die freiwilligen, größtenteils weiblichen Teilnehmer nahmen über zwölf Wochen zweimal wöchentlich an einer Tanzintervention teil, bei denen sie einfache Tanzschritte lernten. Im Vortest-Nachtest-Vergleich zeigte sich, dass sich die Depression und die Behinderungen am Ende der Intervention signifikant verringerten und die körperliche Funktionsfähigkeit sich deutlich erhöhte.

\section{Musik, Gesang und Bewegung bei Müttern mit postnataler Depression}

Die letzte Studie untersuchte die Auswirkung einer fünfwöchigen Mutter-Kind-Gruppentherapie, in der Musik, Gesang und Bewegung bei Müttern mit postnataler Depression eingesetzt wurden. Ziel der Intervention war, die dyadische MutterKind-Intersubjektivität und Gruppenintersubjektivität aufzubauen (Van Puyvelde et al. 2014). Die mikroanalytische Auswertung zeigte, dass sich von der ersten bis zur letzten Sitzung die intersubjektiven Momente mehr als verdoppelten und sich die intersubjektive Zeit verdreifachte. Darüber hinaus schienen die Mütter von der lebhaften Atmosphäre zu profitieren, die ihnen ein Gefühl der Lebendigkeit, Vitalität und Fröhlichkeit zu vermitteln schien.

\section{Schlussfolgerung}

Das Cochrane Review von Meekums und Kolleginnen (2014) bietet eine wichtige Bestandsaufnahme zur Evidenzlage der Tanz-, Bewegungstherapie bei Depression. Um den strengen Prüfungsstandards zur Evidenzbasierung zu genügen, sind größere, methodisch hochwertige Studien nötig. Die manualisierte körperpsychotherapeutische Intervention (Papadopoulos/Röhricht 2014) belegt die Behandlungstreue nach Manualen, was eine wichtige Voraussetzung zur Evidenzbasierung ist (Coleman/Macintosh 2015). Murrock und Graor's Interventionsstudie (2014) weist auf das therapeutische Potential von Tanz bei Depression älterer Menschen hin. Zudem konnte eine dyadische Kurzintervention mit depressiven Müttern die Mutter-Kind-Intersubjektivität verbessern. Zukünftig bedarf es weiterer methodisch hochwertiger Studien zur Evidenzbasierung.

\section{Literatur}

Coleman, K., Macintosh, H. B. (2015): Art and evidence: balancing the discussion on arts- and evidence-based practices with traumatized children. Journal of Child \& Adolescent Trauma 8(1), 21-31, http://dx.doi.org/10.1007/s40653-015-0036-1

Das Cochrane Zentrum (2015): Systematische Übersichtsarbeiten der Cochrane Library. In: www.cochrane.de/de/systematische-uebersichtsarbeiten, 29.3.2015

Meekums, B., Karkou, V., Nelson, E. A. (2015): Dance movement therapy for depression (review). Cochrane Database of Systematic Reviews 2, 1-56, http://dx.doi.org/10.1002/14651858.CDoo9895.pub2

Murrock, C.J., Graor, C. H. (2014): Effects of dance on depression, physical function, and disability in underserved adults. Journal of Aging and Physical Activity 22(3), 380-385, http://dx.doi.org/10.1123/ JAPA.2013-0003

Papadopoulos, N. L. R., Röhricht, F. (2014): An investigation into the application and processes of manualised group body psychotherapy for depressive disorder in a clinical trial. Body, Movement and Dance in Psychotherapy 9(3), 167-180, http:// dx.doi.org/10.1080/17432979.2013.847499 
Röhricht, F., Papadopoulos, N., Priebe, S. (2013): An exploratory randomized controlled trial of body psychotherapy for patients with chronic depression. Journal of Affective Disorders 151, 85-91, http://dx.doi.org/10.1016/j.jad.2013.05.056
Van Puyvelde, M., Rodrigues, H., Loots, G., De Coster, L., Du Ville, K., Matthijs, L., Pattyn, N. (2014): Shall we dance? Music as a port of entrance to maternalinfant intersubjectivity in a context of postnatal depression. Infant Mental Health Journal 35(3), 220-232, http://dx.doi.org/10.1002/imhj.21431

\section{Die Autorin}

\section{Dr. Iris Bräuninger}

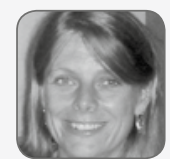

Wissenschaftliche Mitarbeiterin Psychiatrische Universitätsklinik Zürich, Dozentin im Masterstudiengang Tanztherapie, Autonome Universität Barcelona,

Supervisorin und Lehrtherapeutin des deutschen und spanischen Berufsverbandes (BTD, ADMTE), Kestenberg Bewegungsanalytikerin, Psychotherapie (ECP), Private Praxis für Supervision und Therapie.
Dr. Iris Bräuninger

Wissenschaftliche Mitarbeiterin

Psychiatrische Universitätsklinik Zürich

Direktion Pflege, Therapien und Soziale Arbeit

Forschung und Entwicklung

Lenggstr. 31 | $\mathrm{CH}-8032$ Zürich

Tel. $0041-(0) 443842717$

0041-(0)774422676

iris.braeuninger@puk.zh.ch oder dancetherapy@mac.com 